\title{
On the Achievable Rates for the Return-Link of Multi-Beam Satellite Systems Using Successive Interference Cancellation
}

\author{
Vincent Boussemart*, Matteo Berioli*, Francesco Rossetto*, Michael Joham ${ }^{\dagger}$ \\ * Institute of Communications and Navigation, German Aerospace Center (DLR) \\ Oberpfaffenhofen, 82234 Weßling, Germany \\ $\dagger$ Faculty for Electrotechnic and Communication Technology, Methods for Signal Processing \\ Munich University of Technology (TUM), München, Germany \\ E-mail: \{vincent.boussemart, matteo.berioli, francesco.rossetto\}@dlr.de \\ johametum.de
}

\begin{abstract}
This paper investigates the theoretically reachable rates in the return-link of multi-user multi-beam satellite systems. The return-link can actually be analyzed by means of multipleinput multiple-output signal processing techniques. While MIMO inspired multi-user detection for the return-link of satellite systems has been partly investigated in the past, a fundamental look at the achievable gains by these advanced signal processing techniques for satellite communications is missing. Depending on the number of colors used in the system, the available bandwidth per beam can be traded off against the level of co-channel interference. This paper explores the fundamental limits dictated by information theory to the communication rates on satellite multi-beam scenarios, and the impact of a realistic beam patterns on these data rates.
\end{abstract}

\section{INTRODUCTION}

Multi-beam satellite systems are one of the most important and effective ways to increase satellite capacity today [1]. Their principle consists in the combination of signals coming from different antennas on the satellite reflector into multiple beams. Therefore, each beam can be regarded as an antenna with a specially crafted radiation pattern, which is normally designed so as to minimize the spillover into other beams. This concept exploits the spatial separation of the users on ground: if the ground terminals are sufficiently distant, the only beam with a relevant gain is the desired one.

One of the fundamental trade-offs in multi-beam system design is the choice of the number of employed frequencies $K$. The total bandwidth is divided into $K$ channels and each beam is assigned to one of these channels. Of course, the lower $K$, the higher the bandwidth per beam but also the interference from other beams with the same frequency worsens. Therefore there is a clear trade-off between the available bandwidth per beam and the inter-beam interference. For low frequency reuse, the system will be noise-limited, while interference becomes the major limitation when $K$ is small.

The focus of our work is the return-link of a multi-beam system, where multiple users on ground transmit to the satellite. It will be assumed that exactly one user per beam is sending data at any given time, which is the common operation mode in Multi-Frequency - Time Division Multiple Access (MFTDMA) standards, like Digital Video Broadcasting - Return Channel via Satellite (DVB-RCS [2]). This multi-user scenario can be represented as a multiple access channel, and it is known from information theory that the capacity region is attained when a single frequency is adopted and the users are decoded by means of successive interference cancellation multi-user detection [3]. This is in contrast to present day systems, where each beam is decoded independently of the others and $K$ is large enough so at to yield an orthogonal channel allocation (i.e., very low inter-beam interference). Note that such return-link configuration can be regarded as the up-link of a multi-user Multiple-Input Multiple-Output (MIMO) system, where each user has one antenna except for the base station, which has as many antennas as users. For this type of systems, studies on the outage capacity have been performed [4], [5], precoding schemes were proposed and investigated for the forward-link [6], [7], and specific SIC (Successive Interference Cancellation) techniques have been analyzed for the return-link [8], [9].

Previous work [8], [9] has investigated specific multiuser detection schemes. However, a fundamental look at the attainable gains offered by multi-user detection in this context is missing especially taking into account the shape of the beam patterns of current multi-beam systems. The efforts of [8], [9] only propose method that perform well, but it is unclear how much capacity can still be attained in this context if smarter multiuser detection schemes were available. The main contribution of this paper lies in the analysis by means of information theoretical tools of the fundamental performance attainable in the return-link of a satellite system and in the establishment of an upper bound to the attainable gains. While there has been some work that studied the information theoretical capacity of the land mobile satellite channel [10] and there exists extensive and rich literature on the capacity of MIMO terrestrial channels [3], [11], the satellite beam patterns impose a very special structure on the equivalent MIMO channel, which is also dependent on the number of 


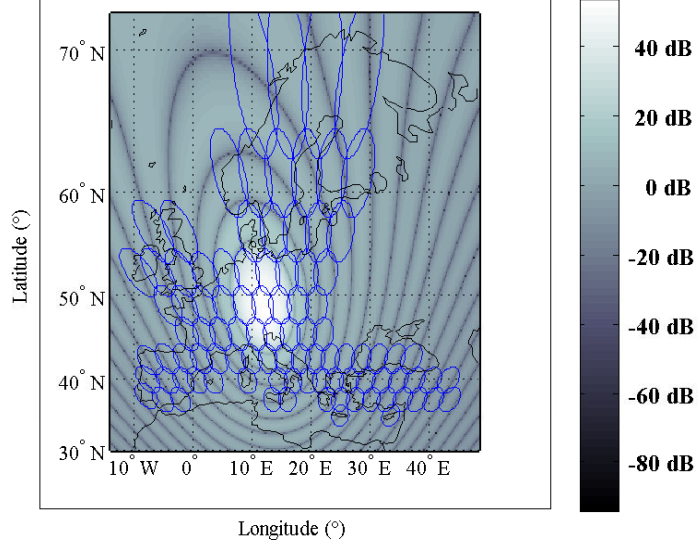

Fig. 1. Satellite system and example of beam radiation pattern. The footprint of the 96 beams considered in the multi-beam satellite scenario are shown. The beam radiation pattern of one single beam is illustrated with the gain in $\mathrm{dB}$. In this example the highlighted beam has the following center coordinates: latitude $48.75^{\circ}$ North and longitude $11.9^{\circ}$ East.

frequencies $K$. Hence, our work bridges the gap between the physical layer studies on multi-user detection for satellites and information theory. Results on the fundamental capacity gains in the return-link of a satellite systems taking into a account a realistic beam pattern are given. Our study explored two main metrics: the sum rate and also proper user scheduling for maximizing the minimum Signal-to-Interference and Noise Ratio (SINR).

The paper is organized as follows. Section II gives an overview of the system model. The following Section III and IV present respectively the computation of the achievable rate and the results obtained for the different scenarios. The conclusions are finally drawn in Section V.

\section{SYSTEM MODEL}

\section{A. Satellite System}

The large majority of today's communication satellites are transparent geostationary (GEO), hence we focused our evaluations on a GEO satellite at $19.2^{\circ}$ East longitude. Moreover, near future satellite communication systems are likely to be operated in Ka-band since this frequency band offers higher data rates in spite of its propagation impairments [1] and hence the adopted frequency is $30 \mathrm{GHz}$.

Modern satellite systems use around one hundred beams; in our evaluations the GEO satellite covers Europe by means of a representative and realistic number of beams: 96 spotbeams. The shape of each spotbeam on the Earth depends on the location of the beam center and on the antenna beam width. From now on we will use the terms spotbeam and beam interchangeably. The beam pattern corresponds to the locations on the Earth where the gain of the satellite antenna is greater than or equal to its maximum gain minus $3 \mathrm{~dB}$. It is assumed that the half-sided beam width is $0.2^{\circ}$ and also that the spotbeams are static: the footprint does not evolve over time. According to the characteristics of the satellite antenna we can derive the radiation pattern of each beam. The radiation pattern corresponds in our case (return-link) to the satellite antenna gain for different satellite open-angles at a frequency $f=30 \mathrm{GHz}$. Fig. 1 shows the radiation pattern of a specific but representative spotbeam computed by means of the equations derived from [6], [12] and given in Appendix B.

To each beam is assigned a frequency band, and the total number of frequency bands is denoted as $K$ also called number of colors or cluster size in the system. If the total available bandwidth in the satellite is $W$, each frequency band is $W / K$ hertz-wide. If we consider as an example the beam of Fig. 1, it is surrounded by other beams. Depending on $K$, the interfering beams, i.e., those using the same frequency, may be far or close to this beam.

We consider in this system model two possible numbers of colors. The multi-beam satellite system can be seen as a set of radio cells (like in conventional cellular networks). Those radio cells are usually considered together in a so-called cluster [13]. The smallest number of colors in conventional satellite systems is $K=3$ because for this value the inter-beam interference is negligible. If the system is noise limited, the system capacity $C$ is approximately proportional to $\left(K A_{\mathrm{b}}\right)^{-1}$, where $A_{\mathrm{b}}$ is the coverage area of a beam [13], and therefore the system capacity can be expected to be three times higher with $K=1$ than with $K=3$ (three times more bandwidth per beam) as long as the inter-beam interference (also denoted as co-channel interference) can be properly handled. The usage of multi-user detection has been recently explored exactly for this purpose, i.e., to enable universal frequency reuse [4], [5].

The satellite terminals are located within the coverage area of the beams. The users are fixed terminals and send data with equal transmit power and identical antenna characteristics toward the satellite. The time is slotted and in each beam and slot one single user is allocated. DVB-RCS, which employs MultiFrequency - Time Division Multiple Access (MF-TDMA) in the return-link, actually complies with this abstraction [2]. Such multi-user multi-beam satellite system can be assumed as a multiple-input (the ground transmitters) multiple-output (the satellite beams) system, i.e., a MIMO system.

\section{B. Channel Matrix}

The notation conventions that are employed in this paper are reported in Appendix A.

As specified in the previous section, the satellite terminals have identical properties. Moreover, in order to focus on the impact of the antenna pattern on the system capacity, clear sky conditions are assumed (thus the channel introduces no fading) and the user antenna is perfectly pointed. When performing the link budget (return- up-link), all terms but two remain constant: the satellite antenna gain (receive gain) and the free space losses which depend directly on the user position.

The former factor is the antenna gain from user $j$ to beam $i$ and it is determined only by the index of the beam and the position of the ground user.

The link budget also depends on the distance $S_{j}$ between user $j$ and the satellite (also called slant range), which introduces a free space loss $L_{\mathrm{FS}, j}$ according to the Friis formula [1]: $L_{\mathrm{FS}, j}=\left(4 \pi S_{j} / \lambda\right)^{2}$ where $\lambda$ is the wavelength. 
We define a coefficient which is normalized according to the maximum gain and the minimum free space loss, i.e., when the user is perfectly located at the beam center and with the smallest slant range. In such a case we have the satellite antenna gain $G_{\max }$ and $L_{\mathrm{FS}, \min }=\min _{j}\left\{L_{\mathrm{FS}, j}\right\}$ the minimum free space loss experienced among the centers of all beams. A given user $j$ will be seen from beam $i$ with a gain $G_{i, j}$ and free space loss $L_{\mathrm{FS}, j}$. The coefficient obtained for that user reflects the modification of the amplitude of the signal. It is denoted $\left|h_{i, j}\right|$ and is equal to:

$$
\left|h_{i, j}\right|=\sqrt{\frac{G_{i, j}}{L_{\mathrm{FS}, j}} \cdot \frac{L_{\mathrm{FS}, \min }}{G_{\max }}}
$$

If $N$ terminals are present, user $j(j \in\{1, \ldots, N\})$ is seen by $N_{\mathrm{b}}$ beams and a beam $i\left(i \in\left\{1, \ldots, N_{\mathrm{b}}\right\}\right)$ is seen by $N$ users. We shall consider the case of one user per beam and hence $N=N_{\mathrm{b}}$. We can compute the different coefficients $h_{i, j}$ for each combination user - beam: we obtain a matrix whose size is $N \times N$ and which corresponds up to a multiplicative constant to the channel matrix $\boldsymbol{H}$. The column $j$ of the matrix describes the channel from user $j$ to all beams (transmitter) and the row $i$ the channel from all users to beam $i$.

The channel matrix $\boldsymbol{H}$ is thus a function of the satellite beam pattern, the position of the users within the beam (different gains) and the distance between the users and the satellite (slant range). In order to simplify the generation of the channel matrix $\boldsymbol{H}$, clear sky conditions (no rain fading and corresponding scintillation increase) and perfect antenna alignment at the user side are also assumed. In addition, perfect Channel State Information (CSI) is assumed at the receiver, whereas the transmitters have no CSI. Finally perfect Interference Cancellation (IC) is also assumed.

\section{Transmission Block Diagram}

We assume that the users transmit a sequence of bits which are modulated and represented at a given time by a vector $\boldsymbol{a}$. The satellite antenna reflector will focus the received signal on the $N_{a}$ feeds, whose signals will be combined to generate the actual beam patterns. All antenna feeds are realistically assumed to have the same antenna pattern. The symbol of the $j$-th user $a_{j}$ received by the $f$-th feed through an equivalent coefficient $b_{f, j}$ that combines the path loss and the feed antenna pattern. These coefficients are collected into a matrix denoted as $\boldsymbol{B}$ (Fig. 2):

$$
\left[\begin{array}{c}
x_{1} \\
\vdots \\
x_{N_{\mathrm{a}}}
\end{array}\right]=\left[\begin{array}{cccc}
b_{1,1} & b_{1,2} & \cdots & b_{1, N} \\
\vdots & \vdots & \ddots & \vdots \\
b_{N_{\mathrm{a}}, 1} & b_{N_{\mathrm{a}}, 2} & \cdots & b_{N_{\mathrm{a}}, N}
\end{array}\right]\left[\begin{array}{c}
a_{1} \\
\vdots \\
a_{N}
\end{array}\right]
$$

which can be written in matrix notation as $\boldsymbol{x}=\boldsymbol{B} \boldsymbol{a}$ where $N_{\mathrm{a}}$ is the number of antennas. ${ }^{1}$

The signal is then handled by a beamformer $\boldsymbol{F}$ which converts the $N_{\mathrm{a}}$ antenna outputs into $N_{\mathrm{b}}$ signals, one per beam. The signal is frequency-shifted, amplified (transparent

\footnotetext{
${ }^{1}$ It has to be noted that the received symbols are assumed to be synchronous at the satellite.
}

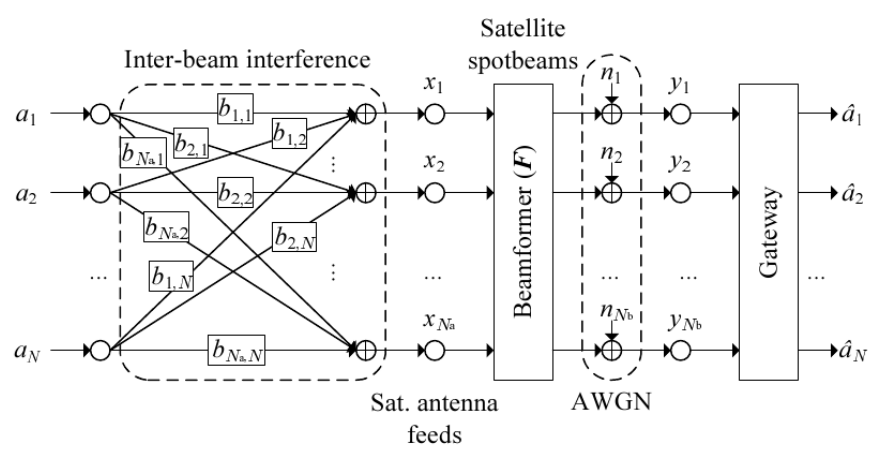

Fig. 2. Multi-beam transmission block diagram. It is composed (from the left to the right) of the inter-beam interference block, satellite antenna feeds, satellite beamformer, the noise block and the gateway. The number of satellite antenna feeds is generally equal or bigger than the number of spotbeams.

payload) and sent back to a processing gateway on ground. Noise is added at the antennas, however we consider the equivalent noise after the beamformer, which is represented by a vector $\boldsymbol{n}$ of size $N_{\mathrm{b}}$ whose $j$-th element is denoted $n_{j}$. We assume Additive White Gaussian Noise (AWGN) with mean value $\mu=0$ and covariance matrix $\sigma^{2} \boldsymbol{I}_{N_{\mathrm{b}}}=N_{0} \boldsymbol{I}_{N_{\mathrm{b}}}$, where $\boldsymbol{I}_{N_{\mathrm{b}}}$ is a $N_{\mathrm{b}} \times N_{\mathrm{b}}$ identity matrix. This assumption would be strictly true if $\boldsymbol{F}$ were unitary but it is nonetheless well satisfied in practice. The signal received by the gateway is:

$$
\boldsymbol{y}=\boldsymbol{F} \boldsymbol{x}+\boldsymbol{n}=\boldsymbol{F}(\boldsymbol{B a})+\boldsymbol{n}
$$

where $y$ is thus a vector of $N_{\mathrm{b}}$ elements. Eq. (3) can be simplified as:

$$
y=H a+n
$$

It is assumed that the relaying of the waveforms to the gateway does not appreciably degrades the noise level and does not alter the equivalent system model of Eq. (3). The gateway estimates the transmitted symbols which are denoted $\hat{\boldsymbol{a}}$. Fig. 2 summarizes the transmission block diagram previously described. It has to be remarked that $\boldsymbol{H}$ will have different properties depending on the cluster size $K$. For $K=3 \boldsymbol{H}$ is almost diagonal, whereas for $K=1$ it is no longer diagonal because of the inter-beam interference.

\section{Phase}

In the previous section the phase is not considered. Actually the channel coefficients are complex numbers thus with an amplitude (obtained with Eq. (1)) and a phase. We assume fixed users on ground. In the return up-link, two phase contributions are added to the signal. The first phase corresponds to the user phase: a given user will be seen by the satellite with the same phase shift across all beams. This means the same phase is applied for all rows in a given column of the channel matrix $\boldsymbol{H}$. The second phase corresponds to the phase shift introduced by the beam forming network (BFN), more particularly it is directly related the BFN characteristics on board. The phase shift between two beams is always the same thus the phase shift between the rows of the channel matrix $\boldsymbol{H}$ is identical for all columns. Once these two phases are applied on the coefficients of the channel matrix, all coefficients have 
different phases. More detailed discussion is addressed in Appendix C.

It should be remarked that if the phases were fully independent and uniformly distributed between 0 and $2 \pi$, the capacity would be higher and thus our computations constitute a lower bound for any system whose phase does not comply with the previous discussion.

\section{ACHiEvable RATES}

As the system model section has shown, it is truly possible to regard the multi-user return-link as a MIMO multiple access channel. Our work wants to investigate the achievable rates in the return-link of a multi-beam satellite system. The signal to noise ratio for $K$ colors and a channel gain $\left|h_{i, i}\right|=1$ is denoted as $\left(E_{\mathrm{s}} / N_{0}\right)_{K}$. The symbol without bracket $E_{\mathrm{s}} / N_{0}$ represents the signal to noise ratio for $K=1$. Since the per user power is kept constant as $K$ is changed, the following relationships hold:

$$
\left(\frac{E_{\mathrm{s}}}{N_{0}}\right)_{K}=K\left(\frac{E_{\mathrm{s}}}{N_{0}}\right)_{1}=K \frac{E_{\mathrm{s}}}{N_{0}}
$$

\section{A. Sum Rates}

On the one hand in conventional systems the user information is decoded based only on the signal of the dedicated beam. Hence, just one out of $N_{\mathrm{b}}$ outputs is employed for any specific user. Moreover all user signals are independently decoded, i.e., interference is not suppressed. The corresponding SingleInput Single-Output (SISO) sum rate is computed using the following formula:

$$
R_{\mathrm{SISO}}=\frac{1}{K} \sum_{j=1}^{N} \log _{2}\left(1+\frac{\left|h_{j, j}\right|^{2}}{\sum_{i \neq j}\left|h_{i, j}\right|^{2}+\left(K E_{\mathrm{s}} / N_{0}\right)^{-1}}\right)
$$

On the other hand when the user signal is handled by the different beams (multiple receivers) and Successive Interference Cancellation is adopted, the resulting Multiple-Input MultipleOutput (MIMO) sum rate in the system is given by [14]:

$$
R_{\mathrm{MIMO}}=\frac{1}{K} \log _{2} \operatorname{det}\left(\boldsymbol{I}_{N_{\mathrm{b}}}+\boldsymbol{C}_{\boldsymbol{n}}^{-1} \boldsymbol{H} \boldsymbol{Q} \boldsymbol{H}^{\mathrm{H}}\right)
$$

where $C_{n}$ and $Q$ are respectively the covariance of the noise and of the transmitted symbols, and $\boldsymbol{H}^{\mathrm{H}}$ is the Hermitian of the channel matrix (conjugate transpose). The symbols are assumed to be independent and to have unit transmit power, thus the matrix $Q$ is equal to $\boldsymbol{I}$. Since the noise is white we obtain:

$$
\boldsymbol{C}_{\boldsymbol{n}}=\sigma^{2} \boldsymbol{I}=\frac{1}{K} \frac{N_{0}}{E_{\mathrm{s}}} \boldsymbol{I}
$$

Between SISO and MIMO there is an intermediate step. It consists into taking advantage of the fact that a given user's signal is present in all beams. Hence the receiver performs maximum ratio combining (MRC) on the signals of the different beams to recover the desired user. The corresponding
Single-Input Multiple-Output (SIMO) rate is calculated by taking into account the following equation:

$$
\begin{aligned}
R_{\text {SIMO }} & =\frac{1}{K} \sum_{j=1}^{N} \log _{2}\left[1+\sum_{i=1}^{N_{\mathrm{b}}} \operatorname{SINR}_{i, j}\right] \\
\operatorname{SINR}_{i, j} & =\frac{\left|h_{i, j}\right|^{2}}{\sum_{k \neq i}\left|h_{k, j}\right|^{2}+\left(K E_{\mathrm{s}} / N_{0}\right)^{-1}}
\end{aligned}
$$

where $\operatorname{SINR}_{i, j}$ is the Signal to Interference and Noise Ratio (SINR) for user $j$ on the signal received in beam $i$. Note that a SIMO approach is useful in a noise-limited regime and for small $K$, since in that case the impact of the interference is negligible and the gain $h_{i, j}$ is significant not only in the desired beam $i=j$. Finally, notice that the sum rate for the SISO approach is the same of the SIMO approach when only the desired beam is considered in the summation of Eq. (10).

Because of the properties of the Hermitian and of the determinant within Eq. (7), and the discussion in Section II-D and Appendix $C$, the phase will have no influence on the resulting rate. The other rates (SISO and SIMO) do not depend on the phase. As a result, the generation of the channel matrix can be done based on Eq. (1) only and therefore $\boldsymbol{H}$ is real.

\section{B. Ordering}

The user rate in the SISO and SIMO approaches is well defined as soon as the channel matrix $\boldsymbol{H}$ is given. On the other hand, the user rates in MIMO can be defined only when a certain detection order is provided. Due to the perfect CSI and IC assumptions, the last user to be decoded in the SIC loop will see only its own column of the channel matrix $\boldsymbol{H}$. The other columns were virtually removed as the impact of the previous users was subtracted. The rate $R_{\mathrm{MIMO}}^{j}$ of user $j$ with SIC can be computed as the difference between the sum rate $R_{\mathrm{MIMO}}^{(N-j+1)}$ of the $N-j+1$ users not yet decoded and sum rate $R_{\mathrm{MIMO}}^{(N-j)}$ of the $N-j$ remaining users after the detection of user $j$ :

$$
\begin{aligned}
R_{\mathrm{MIMO}}^{j} & =\log _{2} \operatorname{det}\left(\boldsymbol{I}_{N_{b}}+\boldsymbol{C}_{\boldsymbol{n}}^{-1} \boldsymbol{H}^{(N-j+1)}\left(\boldsymbol{H}^{(N-j+1)}\right)^{\mathrm{H}}\right) \\
& -\log _{2} \operatorname{det}\left(\boldsymbol{I}_{N_{b}}+\boldsymbol{C}_{\boldsymbol{n}}^{-1} \boldsymbol{H}^{(N-j)}\left(\boldsymbol{H}^{(N-j)}\right)^{\mathrm{H}}\right)
\end{aligned}
$$

where $\boldsymbol{H}^{(N-j)}$ is the matrix composed by the $N-j$ rightmost columns of $\boldsymbol{H}$. By definition, $\boldsymbol{H}^{(N)}=\boldsymbol{H}$ and $\boldsymbol{H}^{0}$ is the empty matrix.

Since the computation of the single user rate is directly related to the columns of the channel matrix $\boldsymbol{H}$, the position of user $j$ in the matrix plays a role. The detection order can be changed by applying a permutation that is to say: $\boldsymbol{H}_{\text {perm }}=$ $\boldsymbol{H} \boldsymbol{P}$ where $\boldsymbol{P}$ is the permutation matrix.

The user rate clearly depends on the specific $\boldsymbol{P}$ chosen out of the $N$ ! possible permutation matrices, while the sum rate remains the same for all permutations. Hence the distribution of the rates between the users changes with $\boldsymbol{P}$. It is thus possible to optimize the ordering, i.e., to find an optimal permutation 
matrix, such that some Quality of Service (QoS) requirements can be fulfilled. We consider the following criterion:

$$
\boldsymbol{P}_{\mathrm{opt}}=\arg \max _{\boldsymbol{P}}\left\{\min _{j}\left(R_{j}(\boldsymbol{P})\right)\right\}
$$

which tries to maximize the minimum rate experienced by the users.

Due to the large number of possible permutations, a brute force enumeration for finding the optimal solution cannot be applied even for moderate number of beams $\left(N_{\mathrm{b}} \geq 10\right)$. Foschini algorithm [15] solves the problem by greedily selecting the user having the best Signal-to-Interference and Noise Ratio (SINR). The user is then removed from the channel matrix and the operation is performed again. Such algorithm returns an ordering and targets the criterion previously defined. The ordering for Minimum Mean Square Error (MMSE) is obtained by considering:

$$
\operatorname{SINR}_{k}^{(j)}=\boldsymbol{h}_{k}^{\mathrm{H}}\left(\boldsymbol{C}_{\boldsymbol{n}}^{-1}+\sum_{i \neq k} \boldsymbol{h}_{i} \boldsymbol{h}_{i}^{\mathrm{H}}\right)^{-1} \boldsymbol{h}_{k}
$$

where $i$ also satisfies $i \notin\left\{k_{1}^{*}, \ldots, k_{j-1}^{*}\right\}$ knowing that:

$$
k_{j}^{*}=\arg \max \left\{\operatorname{SINR}_{k}^{(j)}\right\}, k \in\{1, \ldots, N\} \backslash\left\{k_{1}^{*}, \ldots, k_{j-1}^{*}\right\}
$$

this operation is performed for $j=1, \ldots, N-1$. The term $\boldsymbol{h}_{i}$ designates the $i$-th column of the channel matrix $\boldsymbol{H}$.

\section{Numerical Results}

The $N_{b}=N=96$ spotbeams pattern of Fig. 1 and two cluster sizes, i.e., $K=1$ and $K=3$, were evaluated. The average user rate was computed by dividing the overall sum rate by $N$. The users were placed randomly within the coverage area of each beam, the average user rate is computed over 10000 random sets. In order to correctly compare the two cluster sizes both transmit power and bandwidth were adjusted. As said previously the bandwidth for cluster size 1 is three times the one of cluster size 3. This means there is three times more bandwidth per beam with cluster size 1, but since the per user power is kept constant as $K$ is changed, the SNR for $K=3$ is three times larger than the SNR for $K=1$.

As it can be seen in Fig. 3, cluster size 1 provides more capacity at equivalent $E_{\mathrm{s}} / N_{0}$ than cluster size 3 . The rate for Single Input - Single Output (SISO) considers only the signal sent by user $j$ in its own beam $j(i=j$, diagonal elements of the channel matrix $\boldsymbol{H}$ ) and the interference of the other users $(j \neq i)$ received by the beam (Eq. (6)). For Single Input - Multiple Output (SIMO) the signal sent by user $j$ is considered in all beams (see Eq. (9)). Finally for Multiple Input - Multiple Output (MIMO), the benefit of SIC is evident for $K=1$ at all SNR or also for $K=3$ but for very high SNR (above $20 \mathrm{~dB}$, which do not routinely occur in satellite communications). The level of interference for cluster size 3 is very low: the difference between MIMO and SIMO for high $E_{\mathrm{S}} / N_{0}$ is not huge. Also the three systems are matching for low $E_{\mathrm{s}} / N_{0}$ meaning that the signal level received by the other

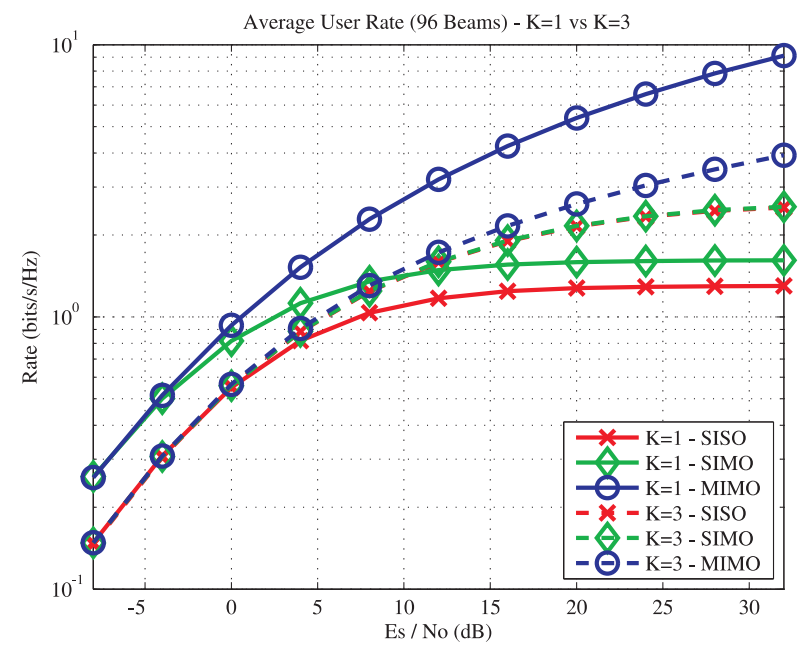

Fig. 3. Average user rate for 96 beams. Rates are compared for $K=1$ and $K=3,10000$ random sets were generated. The following systems are considered: Single Input - Single Output (SISO), Single Input - Multiple Output (SIMO) and Multiple Input - Multiple Output (MIMO).

users for a given beam (interference) is almost negligible. For this reason employing SIMO in cluster size 3 brings no gain over SISO. For cluster size 1 and SISO the user rate is the lowest one: this configuration is highly interference-limited. When switching to SIMO the gain related to the fact that the user signal is considered in all receive beams can be observed and this technique is especially attractive for low SNRs. If the SNR is larger than $0 \mathrm{~dB}$, the system is interference-limited and hence the gain of SIC is visible. At $20 \mathrm{~dB}$ the average user rate for a system with cluster size 3 with SISO and MIMO is about 2.1 and $2.7 \mathrm{bits} / \mathrm{s} / \mathrm{Hz}$, respectively, whereas for cluster size 1 with MIMO it is about $5.4 \mathrm{bits} / \mathrm{s} / \mathrm{Hz}$. This shows how the capacity gain brought by SIC in a satellite system is about almost a factor of 3, compared to a conventional system (SISO $K=3$ without IC) and about a factor of 2 with respect to a classic frequency reuse scheme but with IC. Moreover, the slope of the rate capacity curve at high SNR for MIMO $K=1$ is larger than the slope of the corresponding curve for MIMO $K=3$. The reason is that for stronger frequency reuse the signal of each user is received with higher energy. Indeed, since all beams employ the same frequency, also the beams close to the desired one contribute to the user detection and these beams have a quite strong gain that can improve the system capacity quite remarkably.

The rest of this section deals with the optimization of the single user rates, in particular on the choice of the detection order. Since the number of permutations is $N_{b}$ ! $=N$ !, a smaller scenario was considered with just the reference beam (Fig. 1) and its six surrounding beams with cluster size 1 . We are thus taking into account seven beams, one user per beam at a given time. The minimum single user rate (average value among all random sets) was computed for the following four methods: average, the brute force approach, the Foschini algorithm and finally the worst solution. The average consists into computing the average of the minium user rate over all possible detection 


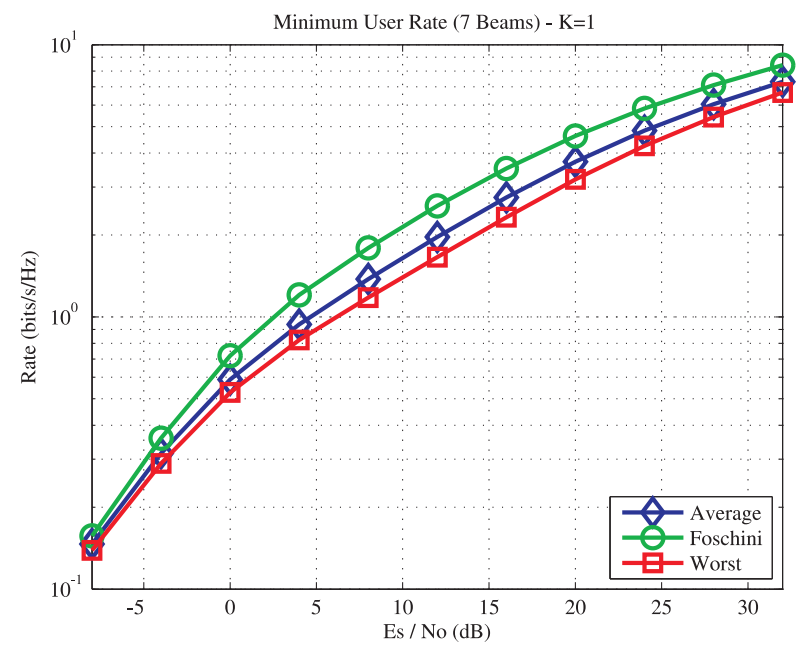

Fig. 4. Minimum rate for 7 beams. The minimum rates are computed for $K=1$. Average rate are compared for different methods: average, brute force and Foschini algorithm.

orders. This strategy represents the performance of a non optimized detection order. The brute force approach resides into computing all possible permutations and into selecting the permutation index satisfying the criterion defined by Eq. (12). For Foschini the algorithm for MMSE is used to find out the optimal ordering. Finally the worst solution aims at selecting the permutation minimizing the minimum user rate.

Fig. 4 shows that the minimum rate is improved compared to an average strategy. With low computation complexity the minimum can be increased by $30 \%$ with respect to a randomly selected permutation and more than $50 \%$ with respect to the permutation that minimizes the minimum rate.

If we want to provide fairness between the users, i.e., minimize the difference of rate between users, this is equivalent to satisfy the criterion defined by $\arg \min _{\boldsymbol{P}}\left\{\operatorname{std}_{j}\left(R_{j}(\boldsymbol{P})\right)\right\}$. As before the same four approaches are considered: average, brute force approach, Foschini algorithm and worst solution. In case $K=1$ Fig. 5 shows that both the brute force approach and the Foschini algorithm decrease the rate difference between users by about a factor of 2 . This time the brute force method performs slightly better than the Foschini algorithm.

\section{Conclusions}

The different numerical results illustrated that interference cancellation does not provide a big capacity increase for cluster size 3. Actually the multi-beam satellite system considered was designed in such a way that co-channel interference is quite low when the cluster size is greater or equal to 3 .

The analysis of the achievable rates has shown that the overall system capacity can be improved by a factor of 2 . A cluster size 1 increases the amount of co-channel interference between the beams but permits however to get more capacity using interference cancellation techniques. The order in which the users are detected plays a significant role since the single user rate changes accordingly. Evaluating the single user rate

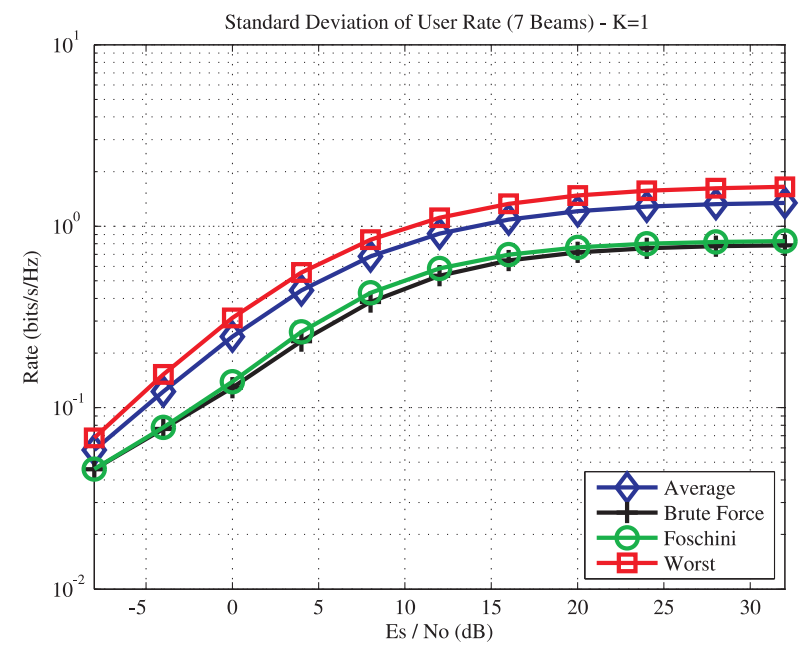

Fig. 5. Standard deviation of the rates for 7 beams. The standard deviation of the rates is computed for $K=1$. Average standard deviations are compared for different methods: average, brute force and Foschini algorithm.

enables to satisfy important Quality of Service (QoS) criteria, like the maximization of the minimum rate.

The performance of the interference cancellation techniques depends of course on the level of interference among the users. As possible future work an important goal would be the resource optimization for $N>N_{\mathrm{b}}$. This would help to take into account the user requirements but also to avoid differences of achievable rate from one time slot to the next one.

\section{ACKNOWLEDGMENT}

This work was in part supported by DFG program 1397 COIN, subcontract Lu1222/1-1. The authors would like to thank Prof. Dr. Erich Lutz and Andreas Gründinger for the useful discussions and hints in preparing this paper.

\section{REFERENCES}

[1] G. Maral and M. Bousquet, Satellite Communications Systems, Fourth Edition, Wiley, 2002.

[2] Digital Video Broadcasting (DVB), Interaction channel for satellite distribution systems, European Standard (Telecommunication series), ETSI EN 301790 V1.5.1, Jan. 2009.

[3] D. Tse and P. Viswanath, Fundamentals of Wireless Communication, Cambridge University Press, 2005.

[4] K. P. Liolis, A. D. Panagopoulos and P.-D. M. Arapoglou, An analytical unifying approach for outage capacity achieved in SIMO and MISO broadband satellite channel configurations, 3rd European Conference on Antennas and Propagation, 2009 (EuCAP 2009), pp. 2911-2915, Mar. 2009.

[5] K. P. Liolis, A. D. Panagopoulos, P. G. Cottis and B. D. Rao, On the applicability of MIMO principle to $10-66 \mathrm{GHz}$ BFWA networks: capacity enhancement through spatial multiplexing and interference reduction through selection diversity, IEEE Transactions on Communications, vol. 57, no. 2, pp. 530-541, Feb. 2009.

[6] M. À. Díaz, N. Courville, C. Mosquera, G. Liva and G. E. Corazza, Non-Linear Interference Mitigation for Broadband Multimedia Satellite Systems, International Workshop on Satellite and Space Communications, IWSSC '07, Salzburg (Austria), pp. 61-65, Sep. 2007.

[7] M. Poggioni, M. Berioli and P. Banelli, BER Performance of Multibeam Satellite Systems with Tomlinson Harashima Precoding in IEEE International Conference on Communications 2009, ICC '09, IEEE, pp. 1-6, Dresden (Germany), June 2009. 
[8] J. P. Millerioux, M. L. Boucheret, C. Bazile and A. Ducasse, Iterative interference cancellation and channel estimation in multibeam satellite systems, International Journal of Satellite Communications and Networking, Wiley, vol. 25, no. 3, pp. 263-283, Mar. 2007.

[9] Novel Intra-System Interference Mitigation Techniques \& Technologies for Next Generations Broadband Satellite Systems, ESTEC Contract No. 18070/04/NL/US, Final report, 2005.

[10] G. Alfano, A. De Maio, and A. M. Tulino, A Theoretical Framework for LMS MIMO Communication Systems Performance Analysis, IEEE Transactions on Information Theory, vol. 56, no. 11, pp. 5614-5630, Nov. 2010.

[11] A. Goldsmith, S. A. Jafar, N. Jindal and S. Vishwanath, Capacity limits of MIMO channels, IEEE Journal on Selected Areas in Communications, vol. 21, no. 5, pp. 684-702, June 2003.

[12] R. E. Collin, Antennas and Radiowave Propagation, International Student Edition, 1985

[13] E. Lutz, M. Werner, and A. Jahn, Satellite Systems for Personal and Broadband Communications, First Edition, Springer-Verlag, 2000.

[14] İ. E. Telatar, Capacity of Multi-antenna Gaussian Channels, Bell Labs Technical Memorandum, June 1995.

[15] G. J. Foschini, G. D. Golden, R. A. Valenzuela and P. W. Wolniansky, Simplified Processing for High Spectral Efficiency Wireless Communication Employing Multi-Element Arrays, IEEE Journal on Selected Areas in Communications, vol. 17, no. 11, pp. 1841-1852, Nov. 1999.

[16] C. Antón-Haro, P. Svedman, M. Bengtsson, A. Alexiou and A. Gameiro, Cross-Layer Scheduling for Multi-User MIMO Systems, IEEE Communications Magazine, pp. 39-45, Sep. 2006.

\section{APPENDIX}

\section{A. Notations}

In this paper the following notations are used:

- Vectors and matrices are denoted by boldface lowercase and uppercase respectively (e.g. $\boldsymbol{a}$ and $\boldsymbol{A}$ )

- Element $i$ of vector $\boldsymbol{a}: a_{i}$

- Element at row $i$ - column $j$ of matrix $\boldsymbol{A}: a_{i, j}$

- Norm of $a_{i, j}:\left|a_{i, j}\right|$

- $i$-th column vector of matrix $\boldsymbol{A}: \boldsymbol{a}_{i}$

- All rows, columns $i$ to $j$ of $\boldsymbol{A}: \boldsymbol{A}_{:, i: j}$

\section{B. Antenna Pattern}

The beams of the satellite can be considered as paraboloidal reflector antennas. The radiation pattern of such antennas was derived from [12], [6]. The next formula permits to compute the gain for different satellite open-angles:

$$
G(u)=G_{\max } \cdot \alpha\left(2 \frac{J_{1}(u)}{u}+\frac{T}{1-T} 2^{p+1} p ! \frac{J_{p+1}(u)}{u^{p+1}}\right)^{2}
$$

where $J_{i}$ is the Bessel function of the first kind and of order $i, G_{\max , \mathrm{dB}}=53.23 \mathrm{~dB}, \alpha=\frac{(1-T)(p+1)}{(1-T)(p+1)+T}$, and $u=\left(\frac{\pi d_{\mathrm{a}}}{\lambda}\right) \sin \theta . d_{\mathrm{a}}$ is the diameter of the antenna (reflector), $\lambda$ is the wavelength and $\theta$ represents the open-angle of interest. In this paper we considered the following typical values: an antenna efficiency $\eta=0.6$, a frequency $f=30 \mathrm{GHz}$, an aperture edge taper $T=20 \mathrm{~dB}$, and the rate at which the aperture field decreases is managed by the coefficient $p(p=2)$.

\section{Phase in Channel Matrix}

The first phase shift in the return up-link corresponds to the user phase. The user phase corresponds to the phase applied on the signal between each user and the satellite. Actually the distance between user $j$ and the satellite is constant, across all beams, but is different for every user. In that case the same phase is inserted on all rows of a given column $j$ of the channel matrix. We define the column phase matrix as:

$$
\phi_{c}=\operatorname{diag}\left\{\mathrm{e}^{\mathrm{j} \theta_{1}}, \mathrm{e}^{\mathrm{j} \theta_{2}}, \cdots, \mathrm{e}^{\mathrm{j} \theta_{N}}\right\}
$$

This resulting channel matrix is obtained by:

$$
\boldsymbol{H}_{\phi_{\mathrm{c}}}=\boldsymbol{H} \boldsymbol{\phi}_{\mathrm{c}}
$$

When looking at Eqs. (6), (9) and (7) the phase has an impact on the MIMO system only. In that case we have:

$$
\begin{gathered}
R_{\phi_{\mathrm{c}}}=\log _{2} \operatorname{det}\left(\boldsymbol{I}_{N_{\mathrm{b}}}+\boldsymbol{C}_{n}^{-1} \boldsymbol{H}_{\boldsymbol{\phi}_{\mathrm{c}}} \boldsymbol{H}_{\boldsymbol{\phi}_{\mathrm{c}}}^{\mathrm{H}}\right) \\
\boldsymbol{H}_{\boldsymbol{\phi}_{\mathrm{c}}} \boldsymbol{H}_{\boldsymbol{\phi}_{\mathrm{c}}}^{\mathrm{H}}=\boldsymbol{H} \boldsymbol{\phi}_{\mathrm{c}}\left(\boldsymbol{H} \boldsymbol{\phi}_{\mathrm{c}}\right)^{\mathrm{H}}=\boldsymbol{H} \boldsymbol{\phi}_{\mathrm{c}} \boldsymbol{\phi}_{\mathrm{c}}^{\mathrm{H}} \boldsymbol{H}^{\mathrm{H}}
\end{gathered}
$$

We finally have:

$$
R_{\phi_{\mathrm{c}}}=\log _{2} \operatorname{det}\left(\boldsymbol{I}_{N_{\mathrm{b}}}+\boldsymbol{C}_{n}^{-1} \boldsymbol{H} \boldsymbol{H}^{\mathrm{H}}\right)=R_{\mathrm{MIMO}}
$$

The phase applied on each column of the channel matrix $\boldsymbol{H}$ thus has no impact on the achievable rate.

In the same way the beam phase corresponds to the phase applied on the signal for each beam. Here the phase shift between user $j$ and beam $i$ depends on the BFN, so it is different from user $j$ - beam $i^{\prime}$, but it is constant for every user. In that case the phase shift between the beams is always the same, i.e., the difference of phase between the rows of the channel matrix is the same for each column $j$. We define the row phase matrix as:

$$
\phi_{\mathrm{r}}=\operatorname{diag}\left\{\mathrm{e}^{\mathrm{j} \theta_{1}}, \mathrm{e}^{\mathrm{j} \theta_{2}}, \cdots, \mathrm{e}^{\mathrm{j} \theta_{N_{\mathrm{b}}}}\right\}
$$

The new channel matrix is computed using the following equation:

$$
\boldsymbol{H}_{\phi_{\mathrm{r}}}=\phi_{\mathrm{r}} \boldsymbol{H}
$$

As before the phase has an impact on the MIMO system only, we have:

$$
\begin{gathered}
R_{\phi_{\mathrm{r}}}=\log _{2} \operatorname{det}\left(\boldsymbol{I}_{N_{\mathrm{b}}}+\boldsymbol{C}_{n}^{-1} \boldsymbol{H}_{\phi_{\mathrm{r}}} \boldsymbol{H}_{\boldsymbol{\phi}_{\mathrm{r}}}^{\mathrm{H}}\right) \\
\left.\operatorname{det}\left(\boldsymbol{I}_{N_{\mathrm{b}}}+\boldsymbol{C}_{n}^{-1} \boldsymbol{H}_{\phi_{\mathrm{r}}} \boldsymbol{H}_{\boldsymbol{\phi}_{\mathrm{r}}}^{\mathrm{H}}\right)=\operatorname{det}\left(\boldsymbol{I}_{N_{\mathrm{b}}}+\boldsymbol{C}_{n}^{-1} \boldsymbol{\phi}_{\mathrm{r}} \boldsymbol{H}\left(\boldsymbol{\phi}_{\mathrm{r}} \boldsymbol{H}\right)^{\mathrm{H}}\right)\right)
\end{gathered}
$$

The following property on determinants is particularly useful:

$$
\operatorname{det}(\boldsymbol{I}+\boldsymbol{A} \boldsymbol{B})=\operatorname{det}(\boldsymbol{I}+\boldsymbol{B} \boldsymbol{A})
$$

It usage enables to write:

$\operatorname{det}\left(\boldsymbol{I}_{N_{\mathrm{b}}}+\boldsymbol{C}_{n}^{-1} \boldsymbol{H}_{\boldsymbol{\phi}_{\mathrm{r}}} \boldsymbol{H}_{\boldsymbol{\phi}_{\mathrm{r}}}^{\mathrm{H}}\right)=\operatorname{det}\left(\boldsymbol{I}_{N_{\mathrm{b}}}+\boldsymbol{C}_{n}^{-1} \boldsymbol{\phi}_{\mathrm{r}}^{\mathrm{H}} \boldsymbol{\phi}_{\mathrm{r}} \boldsymbol{H} \boldsymbol{H}^{\mathrm{H}}\right)$

We finally have:

$$
R_{\phi_{\mathrm{r}}}=\log _{2} \operatorname{det}\left(\boldsymbol{I}_{N_{\mathrm{b}}}+\boldsymbol{C}_{n}^{-1} \boldsymbol{H} \boldsymbol{H}^{\mathrm{H}}\right)=R_{\mathrm{MIMO}}
$$

The phase applied on each row of the channel matrix $\boldsymbol{H}$ has also no impact on the achievable rate.

When looking at the previous properties, if a realistic phase should be included in the channel matrix $\boldsymbol{H}$, then a particular phase for each user and the same phase shift between the beams should be added. However, because of the previous discussion, this does not have an influence on the achievable rate. For this reason the channel matrix in this scenario contains only real numbers. 